\title{
Mean-Field Approximation, Convex Hierarchies, and the Optimality of Correlation Rounding: A Unified Perspective*
}

\author{
Vishesh Jain \\ Frederic Koehler \\ visheshj@mit.edu \\ fkoehler@mit.edu \\ Massachusetts Institute of Technology. Department of \\ Mathematics. \\ Cambridge, MA, USA
}

\author{
Andrej Risteski \\ risteski@mit.edu \\ Massachusetts Institute of Technology. Department of \\ Mathematics and IDSS. \\ Cambridge, MA, USA
}

\begin{abstract}
The free energy is a key quantity of interest in Ising models, but unfortunately, computing it in general is computationally intractable. Two popular (variational) approximation schemes for estimating the free energy of general Ising models (in particular, even in regimes where correlation decay does not hold) are: (i) the mean-field approximation with roots in statistical physics, which estimates the free energy from below, and (ii) hierarchies of convex relaxations with roots in theoretical computer science, which estimate the free energy from above. We show, surprisingly, that the tight regime for both methods to compute the free energy to leading order is identical.

More precisely, we show that the mean-field approximation to the free energy is within $O\left(\left(n\|J\|_{F}\right)^{2 / 3}\right)$ of the true free energy, where $\|J\|_{F}$ denotes the Frobenius norm of the interaction matrix of the Ising model. This simultaneously subsumes both the breakthrough work of Basak and Mukherjee, who showed the tight result that the mean-field approximation is within $o(n)$ whenever $\|J\|_{F}=o(\sqrt{n})$, as well as the work of Jain, Koehler, and Mossel, who gave the previously best known non-asymptotic bound of $O\left(\left(n\|J\|_{F}\right)^{2 / 3} \log ^{1 / 3}\left(n\|J\|_{F}\right)\right)$. We give a simple, algorithmic proof of this result using a convex relaxation proposed by Risteski based on the Sherali-Adams hierarchy, automatically giving sub-exponential time approximation schemes for the free energy in this entire regime. Our algorithmic result is tight under Gap-ETH.

We furthermore combine our techniques with spin glass theory to prove (in a strong sense) the optimality of correlation rounding, refuting a recent conjecture of Allen, O'Donnell, and Zhou. Finally, we give the tight generalization of all of these results to $k$-MRFs, capturing as a special case previous work on approximating MAX$k$-CSP.
\end{abstract}

${ }^{*}$ V.J. is partially supported by NSF CCF 1665252 and DMS-1737944 and ONR N0001417-1-2598. F.K. is partially supported by NSF Large CCF-1565235 and Ankur Moitra's David and Lucile Packard Fellowship.

Permission to make digital or hard copies of all or part of this work for personal or classroom use is granted without fee provided that copies are not made or distributed for profit or commercial advantage and that copies bear this notice and the full citation on the first page. Copyrights for components of this work owned by others than the author(s) must be honored. Abstracting with credit is permitted. To copy otherwise, or republish, to post on servers or to redistribute to lists, requires prior specific permission and/or a fee. Request permissions from permissions@acm.org.

STOC '19, fune 23-26, 2019, Phoenix, AZ, USA

(c) 2019 Copyright held by the owner/author(s). Publication rights licensed to ACM ACM ISBN 978-1-4503-6705-9/19/06 . .\$15.00

https://doi.org/10.1145/3313276.3316299

\section{CCS CONCEPTS}

- Mathematics of computing $\rightarrow$ Markov networks; • Theory of computation $\rightarrow$ Rounding techniques.

\section{KEYWORDS}

Variational methods, graphical models, mean-field approximation, correlation rounding, convex hierarchies

\section{ACM Reference Format:}

Vishesh Jain, Frederic Koehler, and Andrej Risteski. 2019. Mean-Field Approximation, Convex Hierarchies, and the Optimality of Correlation Rounding: A Unified Perspective. In Proceedings of the 51st Annual ACM SIGACT Symposium on the Theory of Computing (STOC '19), fune 23-26, 2019, Phoenix, AZ, USA. ACM, New York, NY, USA, 11 pages. https://doi.org/10.1145/ 3313276.3316299

\section{INTRODUCTION}

One of the most widely studied probabilistic models in statistical physics and machine learning is the Ising model, which is a probability distribution on the hypercube $\{ \pm 1\}^{n}$ of the form

$$
P[X=x]:=\frac{1}{Z} \exp \left(\sum_{i<j} J_{i, j} x_{i} x_{j}\right)=\frac{1}{Z} \exp \left(\frac{1}{2} x^{T} J x\right),
$$

where $\left\{J_{i, j}\right\}_{i, j \in\{1, \ldots, n\}}$ are the entries of an arbitrary real, symmetric matrix with zeros on the diagonal. The distribution $P$ is also referred to as the Boltzmann distribution or Gibbs measure. The key quantity of interest is the normalizing constant

$$
Z:=\sum_{x \in\{ \pm 1\}^{n}} \exp \left(\sum_{i<j} J_{i, j} x_{i} x_{j}\right)
$$

known as the partition function of the Ising model, and its logarithm, $\mathcal{F}:=\log Z$, known as the free energy. The reason these are important is that one can easily extract from them many other quantities of interest, most notably the values of the marginals (probabilities like $P\left[X_{i}=x_{i}\right]$ ), phase transitions in the behavior of the distribution (e.g. existence of long-range correlations), and many others.

Although originally introduced in statistical physics, Ising models and their generalizations have also found a wide range of applications in many different areas like statistics, computer science, combinatorics and machine learning (see the references and discussion in $[6,7,35])$. Consequently, various different algorithmic and 
analytic approaches to computing and/or approximating the free energy have been developed.

We should note at the outset that the partition function is both analytically and computationally intractable: closed form expressions for the partition function are extremely hard to derive (even for the Ising model on the standard 3-dimensional lattice), and approximating the partition function multiplicatively is NP-hard, even in the case of graphs with degrees bounded by a small constant (see [32]).

Nevertheless, there are a plethora of approaches to approximating the partition function - both for the purposes of deriving structural results, and for designing efficient algorithms. A major group of approaches consists of the so-called variational methods, which proceed by writing a variational expression for the free energy, and then modifying the resulting optimization problem in some way so as to make it tractable. More concretely, one can write the free energy using the Gibbs variational principle as

$$
\mathcal{F}=\max _{\mu}\left[\sum_{i<j} J_{i j} \mathbb{E}_{\mu}\left[X_{i} X_{j}\right]+H(\mu)\right],
$$

where $\mu$ ranges over all probability distributions on the Boolean hypercube. This can be seen by noting that

$$
\mathrm{KL}(\mu \| P)=\mathcal{F}-\sum_{i<j} J_{i j} \mathbb{E}_{\mu}\left[X_{i} X_{j}\right]-H(\mu)
$$

and recalling that $\mathrm{KL}(\mu \| P) \geq 0$ with equality if and only if $\mu=P$.

Of course, the polytope of distributions $\mu$ is intractable to optimize over. Two popular approaches for handling this are:

- Mean-field approximation: instead of optimizing over all distributions, one optimizes over product distributions, thereby obtaining a lower bound on $\mathcal{F}$. In other words, we define the (naive mean-field) variational free energy by

$$
\mathcal{F}^{*}:=\max _{x \in[-1,1]^{n}}\left[\sum_{i<j} J_{i j} x_{i} x_{j}+\sum_{i} H\left(\frac{x_{i}+1}{2}\right)\right] .
$$

Indeed, if $\bar{x}=\left(\bar{x}_{1}, \ldots, \bar{x}_{n}\right)$ is the optimizer in the above definition, then the product distribution $v$ on the Boolean hypercube with the $i^{t h}$ coordinate having expectation $\bar{x}_{i}$ minimizes $\mathrm{KL}(\mu \| P)$ among all product distributions $\mu$.

This approach originated in the physics literature where it was used to great success in several cases, but from the point of view of algorithms it is a priori problematic: it's not clear this problem is any easier to solve, as the resulting optimization problem is highly non-convex.

- Moment-based convex relaxations: instead of optimizing over distributions, one optimizes over a "relaxation" (enlarging) of the polytope of distributions, thereby obtaining an upper bound on $\mathcal{F}$. There are systematic ways to do this, giving rise to hierarchies of convex relaxations (see, e.g. [5]). This approach is very natural and common in theoretical computer science, since the optimization problem is convex, hence efficiently solvable, although quantifying the quality of the relaxation is usually more difficult.

A priori these two approaches seem unrelated - indeed, the way they modify the variational problem is almost opposite. In this paper, we provide a unified perspective on these two approaches: for example, we show that the tight parameter regime where meanfield approximation and Sherali-Adams based approaches (even for classical MAX- $k$-CSP) give nontrivial guarantees is identical.

More precisely, we prove the following results:

(1) Simple and optimal mean-field bounds via rounding: We obtain the optimal bounds on the quality of the meanfield approximation in a simple and elegant way. In particular, we show that there is a simple rounding procedure which directly extracts a product distribution from the true Gibbs measure, and whose output is easy to analyze. More precisely, a recent result due to Jain, Koehler, and Mossel [20] proves that the mean-field approximation to $\mathcal{F}$ is within an additive error $^{1}$ of $O\left(n^{2 / 3}\|J\|_{F}^{2 / 3} \log ^{1 / 3}\left(n\|J\|_{F}\right)\right)$. We improve this and show:

Theorem 1.1. Fix an Ising model $J$ on $n$ vertices. Then,

$$
\mathcal{F}-\mathcal{F}^{*} \leq 3 n^{2 / 3}\|J\|_{F}^{2 / 3}
$$

We note that [20] prove this inequality is tight up to constants. This also recovers the result of Basak and Mukherjee [6], which shows the error is $o(n)$ when $\|J\|_{F}^{2}=o(n)$. The technique also gives a structural result showing that certain conditional marginals are approximate fixpoints of the mean-field equations. The full results are in Section 4.

(2) Subexponential algorithms for approximating $\mathcal{F}$ up to the computational intractability limit: Our proof of the above theorem is algorithmic, except that it assumes access to the true Gibbs measure. To fix this, we instead apply our rounding scheme to a convex relaxation proposed by Risteski [31] based on the Sherali-Adams hierarchy. The algorithm we get as a result runs in subexponential time so long as $\|J\|_{F}^{2}=o(n)$; this condition for subexponentiality is tight under Gap-ETH. More precisely:

THeOREM 1.2. We can approximate $\mathcal{F}$ up to an additive factor of o(n) in time $2^{o(n)}$ if $\|J\|_{F}^{2}=o(n)$. Moreover, we can also output a product distribution achieving this approximation. On the other hand, for $\|J\|_{F}^{2}=\Theta(n)$, it is Gap-ETH-hard to approximate $\mathcal{F}$ up to an additive factor of o $(n)$ in subexponential time.

We also describe how to accelerate the algorithm on dense graphs using random subsampling. The full results are in Section 7.

(3) Optimality of correlation rounding: The rounding we use in the proof of the above theorems relies crucially on the correlation rounding technique introduced in [5]. This procedure was designed specifically to tackle dense and spectrally well-behaved instances of constraint satisfaction problems, as well as to derive subexponential algorithms for unique games. In order to better understand the efficacy of correlation rounding, Allen, O’Donnell, and Zhou [3] introduced a conjecture on the number of variables one needs to condition on in an arbitrary distribution, in order to guarantee that the remaining pairs of variables have average covariance at

${ }^{1}$ Here, $\|J\|_{F}:=\sqrt{\sum_{i, j} J_{i, j}^{2}}$ is the Frobenius norm of the matrix $J$. 
most $\epsilon$. The current best result of Raghavendra and Tan [30] gives a bound of $O\left(1 / \epsilon^{2}\right)$; [3] conjectured that this can be decreased to $O(1 / \epsilon)$. We refute this conjecture in essentially the strongest possible sense. Namely, we show:

Theorem 1.3. There exists an absolute constant $C>0, a$ sequence of pairs $\left(t_{n}, n\right)$ going to infinity, and a family of probability distributions (the SK spin glass) such that for any set $T$ with $|T| \leq t_{n}$,

$$
\mathbb{E}_{(i, j) \sim\left(\begin{array}{c}
{[n]} \\
2
\end{array}\right)}\left[\left|\operatorname{Cov}\left(X_{i}, X_{j}\right)\right| \mid\left(X_{k}\right)_{k \in T}\right] \geq \frac{C}{\sqrt{t_{n}}} .
$$

We prove this theorem by combining our techniques with rigorous results on the Sherrington-Kirkpatrick spin glass. The full results are in Section 6.

(4) Generalization of all results to $k$-MRFs: We give natural and tight generalizations of these results to order $k$ Markov Random Fields. In general, we show that the tight regime for $o(n)$ additive error for both mean-field and sub-exponential time algorithms (under Gap-ETH) is $\|J\|_{F}^{2}=o\left(n^{3-k}\right)$, and show tightness of the higher-order correlation rounding guarantee. Full results appear in Section 5.

\section{BACKGROUND AND RELATED WORK}

\subsection{The Mean-Field Approximation}

Owing to its simplicity, the (naive) mean field approximation has long been used in statistical physics (see [29] for a textbook treatment) and also in Bayesian statistics [4, 23, 35], where it is one of the prototypical examples of a variational method. It has the attractive property that it always gives a lower bound for the free energy.

The critical points of $\mathcal{F}^{*}$ have a fixpoint interpretation as the solutions to the mean-field equation, $x=\tanh ^{\otimes n}(J x)$. However, iterating this equation is known to converge to the mean-field solution only in high-temperature regimes such as Dobrushin uniqueness; as soon as we leave this regime, the iteration may fail to converge to the optimum even in simple models (Curie-Weiss) - see [20]. In contrast, Eldan and Gross [13] established a structural result, without relying on a high-temperature assumption, showing that the Gibbs measure can be decomposed into approximate fixpoints of the mean-field equations. In the full version of this paper [22], we derive a similar result using the correlation rounding decomposition.

It is well known [14] that the mean field approximation is very accurate for the Curie-Weiss model (the Ising model on the complete graph) at all temperatures. On the other hand, it is also known [11] that for very sparse graphs like trees of bounded arity, this is not the case. In recent years, considerable effort has gone into bounding the error of the mean-field approximation on more general graphs; we refer the reader to $[6,20]$ for a detailed discussion and comparison of results in this direction. If one only wishes to show that the meanfield approximation asymptotically gives the correct free energy density $\mathcal{F} / n$ and does not care about the rate of convergence, then the breakthrough result is due to Basak and Mukherjee [6], who provided an exponential improvement over previous work of Borgs, Chayes, Lovász, Sós, and Vesztergombi [7] to identify the regime where this happens.
THEOREM 2.1 ([6]). Let $\left(J_{n}\right)_{n=1}^{\infty}$ be a sequence of Ising models indexed by the number of vertices. if $\left\|J_{n}\right\|_{F}^{2}=o(n)$, then $\mathcal{F}_{J_{n}}-\mathcal{F}_{J_{n}}^{*}=$ $o(n)$.

This result is tight - there are simple examples of models with $\left\|J_{n}\right\|_{F}^{2}=\Theta(n)$ where $\mathcal{F}_{J_{n}}-\mathcal{F}_{J_{n}}^{*}=\Omega(n)$. On the other hand, if one also cares about the rate of convergence, then this result is not the best known. Here, improving on previous bounds of Borgs et al. [7], Basak and Mukherjee [6], and Eldan [12], it was shown by Jain, Koehler and Mossel [20] that:

Theorem 2.2 ([20]). Fix an Ising model J on $n$ vertices. Then,

$$
\mathcal{F}-\mathcal{F}^{*} \leq 200 n^{2 / 3}\|J\|_{F}^{2 / 3} \log ^{1 / 3}\left(n\|J\|_{F}+e\right) .
$$

As stated earlier, our first main result Theorem 1.1 removes the logarithmic term in Theorem 2.2, thereby completely subsuming both of the theorems stated above. A more general version of this theorem, valid for higher-order Markov random fields on arbitrary finite alphabets, appears in Section 5.

\subsection{Algorithms for Dense Graphs}

At first glance, the condition that $\|J\|_{F}^{2}=o(n)$ may seem a little odd. To demystify it, consider the anti-ferromagnetic Ising model corresponding $^{2}$ to MAX-CUT on a graph with $m$ edges which has $J_{i j}=-\frac{\beta n}{m}$ for each $(i, j) \in E$. If $M$ is the optimum fraction of edges cut, then

$$
\frac{1}{n \beta} \log Z \in\left[M-\frac{1}{\beta}, M+\frac{1}{\beta}\right], \quad\|J\|_{F}^{2}=\Theta\left(\beta^{2} \frac{n^{2}}{m}\right),
$$

so the requirement that $\|J\|_{F}^{2}=o(n)$ is the same as requiring $m=\omega(n)$. In other words, our algorithms operate in the regime where the average degree is super-constant and the objective is to approximate MAX-CUT within factor $(1-\epsilon)$. Thus, they can be viewed as free-energy generalizations of optimization problems on dense graphs.

We briefly survey relevant work on approximation algorithms for dense graphs. The main emphasis in the literature has been on the case when $m=\Theta\left(n^{2}\right)$ for which PTASs have been developed, for instance the weak regularity lemma based algorithm of Frieze and Kannan [16], the greedy algorithms of Mathieu and Schudy [26], and the Sherali-Adams based approach of de la Vega and Mathieu [10]. On the other hand, if $m=\Theta\left(n^{2-\epsilon}\right)$ for any $\epsilon>0$ then no PTAS for even MAX-CUT is possible [9].

The work most relevant to ours is the improved analysis of the Sherali-Adams relaxation due to Yoshida and Zhou [37] based on correlation rounding. Surprisingly, although there are many methods to approximate MAX-CUT when $m=\Theta\left(n^{2}\right)$ as mentioned above, to our knowledge none of the algorithms except for SheraliAdams are guaranteed to give sub-exponential time algorithms down to $m=\omega(n)$; for example, the method of [16] is only subexponential time for $m=\omega(n \log n)$ and this is a fundamental barrier with their technique. This sub-exponential time guarantee for Sherali-Adams in this regime is not explicitly stated in [37] or anywhere else, as far as we are aware, but is straightforward to show even from the older correlation rounding guarantee of [30]

${ }^{2}$ The scaling here is chosen so that if the MAX-CUT is $\gamma n$ edges with $\gamma>1 / 2$, then the two terms in (1) are of the same scale. 
(see Section 7). The correct generalization of this guarantee for MAX- $k$-CSP was essentially pointed out by Fotakis, Lampis, and Paschos in [15] but once again, their algorithm misses the tight regime (achievable by Sherali-Adams) by poly-logarithmic factors. Our result recovers the tight regime (i.e. $\omega\left(n^{k-1}\right)$ constraints) in this setting as well, while also generalizing to the free energy (see Section 7).

For computing the free energy, the two most relevant works are [31] and [20]: the first work does not make any connection to mean-field approximation and proves a slightly weaker guarantee for Sherali-Adams than the current work; the second work uses a regularity based approach to compute the mean-field approximation, and gets similar guarantees to the algorithm of this work but misses the correct sub-exponential time regime by log factors.

\subsection{Correlation Rounding, and a Refutation of the Allen-O'Donnell-Zhou Conjecture}

Let $X_{1}, \ldots, X_{n}$ be a collection of jointly distributed random variables, each of which takes values in $\{ \pm 1\}$. There are two possibilities for such a collection:

- The average covariance of the collection, defined to be $\mathbb{E}_{(i, j) \sim\left(\begin{array}{c}{[n]} \\ 2\end{array}\right)}\left|\operatorname{Cov}\left(X_{i}, X_{j}\right)\right|$, is small.

- The average covariance of the collection is not small: in this case, we expect a random coordinate $X_{j}$ to contain significant information about many of the other random variables in $X_{1}, \ldots, X_{n}$, so that we might intuitively conjecture that conditioning on the random variables $X_{j}$ for all $j$ in a 'small' random subset $T$ of $[n]$ makes the average covariance sufficiently small.

This intuition is indeed true and has been quantitatively formalized by several works in the theoretical computer science community, including $[5,18,30,37]$. We note that similar ideas have appeared independently in the statistical physics literature under the name of 'pinning'; for example, see [19] and references therein, as well as recent work [8].

Theorem 2.3 ([30]). Let $X_{1}, \ldots, X_{n}$ be a collection of $\{ \pm 1\}$-valued random variables, and let $0<\epsilon \leq 1$. Then, for some integer $0 \leq t \leq$ $O\left(1 / \epsilon^{2}\right)$ :

$$
\mathbb{E}_{T \sim\left(\begin{array}{c}
V \\
t
\end{array}\right)} \mathbb{E}_{(i, j) \sim\left(\begin{array}{c}
{[n]} \\
2
\end{array}\right)}\left[\left|\operatorname{Cov}\left(X_{i}, X_{j}\right)\right| \mid\left(X_{k}\right)_{k \in T}\right] \leq \epsilon .
$$

The above theorem is at the heart of the so-called correlation rounding technique for the Sherali-Adams and SOS convex relaxation hierarchies, which has been used to provide state-of-the-art approximation algorithms for many classic NP-hard problems and their variants; we refer the reader to the references above for much more on this. As we will see below, it will also be key to our proof of Theorem 1.1.

Recently, it was conjectured by Allen, O’Donnell and Zhou [3] that the upper bound on $t$ in Theorem 2.3 can be improved significantly. More precisely, they conjectured that:

Conjecture 2.4 (Conjecture A in [3]). Theorem 2.3 holds with $0 \leq t \leq O(1 / \epsilon)$.

Their motivation for this conjecture was twofold:
- On a technical level, the proof of Theorem 2.3 in [30] proceeds by first showing that for some integer $0 \leq t \leq O\left(1 / \epsilon^{2}\right)$

$$
\mathbb{E}_{T \sim\left(\begin{array}{c}
V \\
t
\end{array}\right)} \mathbb{E}_{(i, j) \sim\left(\begin{array}{c}
{[n]} \\
2
\end{array}\right)}\left[\left|I\left(X_{i}, X_{j}\right)\right| \mid\left(X_{k}\right)_{k \in T}\right] \leq \epsilon^{2},
$$

where $I(X, Y)$ denotes the mutual information between $X$ and $Y$, and then using the standard inequality $|\operatorname{Cov}(X, Y)| \leq$ $\sqrt{2 I(X, Y)}$; we will present a generalized version of this proof from [25, 37] later. Essentially, they conjectured that one could surmount the quadratic loss without passing through mutual information.

- From a complexity-theoretic point of view, the best known lower bounds for dense MAX-CSP problems (such as [1, 25]) leave open the possibility that MAX-CUT on $n$ vertices can be computed to within $\epsilon n^{2}$ additive error in time $n^{O(1 / \epsilon)}$, whereas the best known algorithms all require time at least ${ }_{2} O\left(1 / \epsilon^{2}\right)$. If Conjecture 2.4 were true, the running time of the Sherali-Adams based approach would have improved to $n^{O(1 / \epsilon)}$ time for $\epsilon n^{2}\|J\|_{\infty}$ error (which, for dense graphs, is close to matching the lower bound of [25]).

The authors in [3] prove Conjecture 2.4 for the special case when the random variables $X_{1}, \ldots, X_{n}$ are the leaves of a certain type of information flow tree known as the caterpillar graph. In addition, [25] showed a similar improvement for correlation rounding in the MAX $k$-CSP problem, when promised that there exists an assignment satisfying all of the constraints. As described in the introduction (Theorem 1.3), we use ideas from statistical physics to refute Conjecture 2.4 in essentially the strongest possible form by showing that Theorem 2.3 does not hold with $0 \leq t \leq o\left(1 / \epsilon^{2}\right)$.

\section{TECHNICAL TOOLS}

\subsection{Hierarchies of Convex Relaxations}

Computing the free energy of an Ising model has as a special case the problem MAX-QP/MAX-2CSP, because if we let $J_{\beta}=\beta J$ then

$$
\begin{aligned}
\lim _{\beta \rightarrow \infty} \frac{1}{\beta} \log Z\left(J_{\beta}\right) & =\lim _{\beta \rightarrow \infty} \sup _{\mu}\left(\frac{1}{2} \mathbb{E}\left[X^{T} J X\right]+\frac{1}{\beta} H(\mu)\right) \\
& =\max _{x \in\{ \pm 1\}^{n}} x^{T} J x .
\end{aligned}
$$

As with many other problems in combinatorial optimization, this is a maximization problems on the Boolean hypercube, i.e. a problem of the form

$$
\max _{x \in\{ \pm 1\}^{n}} f(x) .
$$

These problems are often NP-hard to solve exactly, but convex hierarchies give a principled way to write down a natural family of convex relaxations which are efficiently solvable and give increasingly better approximations to the true value. First, one re-expresses the problem as an optimization problem over the convex polytope of probability distributions using that

$$
\max _{x \in\{ \pm 1\}^{n}} f(x)=\max _{\mu \in \mathcal{P}\left(\{ \pm 1\}^{n}\right)} \mathbb{E}_{\mu}[f(x)] ;
$$

the advantage of this reformulation is that the objective is now linear in the variable $\mu$. Second, one relaxes $\mathcal{P}\left(\{ \pm 1\}^{n}\right)$ to a larger convex set of pseudo-distributions which are more tractable to optimize over. The tightness of relaxation is controlled by a parameter $r$ (known as the level or number of rounds of the hierarchy); as the 
parameter $r$ increases, the relaxation becomes tighter with the level $n$ relaxation corresponding to the original optimization problem.

Different hierarchies correspond to different choices of the space of pseudo-distributions; two of the most popular are the Sherali-Adams (SA) hierarchy and the Sum-of-Squares (SOS)/Laserre hierarchy. In the Sherali-Adams hierarchy, we define a level $r$ pseudodistribution to be given by the following variables and constraints:

(1) For every $S \subset[n]$ with $|S|=r$, a valid joint distribution $\mu_{S}$ over $\{ \pm 1\}^{S}$.

(2) Compatability conditions, which require that for every $U \subseteq$ [n] with $|U| \leq r$ and every $S, S^{\prime} \subseteq[n]$ with $|S|=\left|S^{\prime}\right|=r$ and $U \subset S \cap S^{\prime},\left.\mu_{S}\right|_{U}=\left.\mu_{S^{\prime}}\right|_{U}$.

Observe that, by linearity, this data defines a unique pseudoexpectation operator ${ }^{3} \tilde{\mathbb{E}}$ from real polynomials of degree at most $r$ to $\mathbb{R}$.

Let $S A_{r}$ denote the set of level $r$-pseudodistributions on the hypercube. Then for $r \geq \operatorname{deg}(f)$, we can write down $\max _{\mu \in S A_{r}} \tilde{\mathbb{E}}_{\mu}[f(x)]$ as a linear program with $2^{r}\left(\begin{array}{c}n \\ r\end{array}\right)$ many variables and a number of constraints which is polynomial in the number of variables. By strong duality for linear programs, we can also think of the value of the level $r S A$ relaxation as corresponding to the best upper bound derivable on $\sup _{\mu} \mathbb{E}_{\mu}[f(x)]$ in a limited proof system, which captures e.g. case analysis on sets of size at most $r$.

In addition to this standard setup, since the variational formulation for $\log Z$ has an entropy term, we will need a proxy for it when we use the Sherali-Adams hierarchy. The particular proxy we will use was introduced by [31] - further details are in Section 7.

\subsection{The Correlation Rounding Theorem}

As mentioned in the introduction, our proof of Theorem 1.1 will depend crucially on the correlation rounding theorem. Here, we present a general higher-order version of this theorem due to Manurangsi and Raghavendra [25], building on previous work of Raghavendra and Tan [30], and Yoshida and Zhou [37].

Definition 3.1. The multivariate total correlation of a collection of random variables $X_{1}, \ldots, X_{n}$ is defined to be

$$
C\left(X_{1} ; \cdots ; X_{n}\right)=\mathbf{K L}\left(\mu\left(X_{1}, \ldots, n\right) \| \mu\left(X_{1}\right) \times \cdots \times \mu\left(X_{n}\right)\right) .
$$

From the definition of KL divergence, it follows that

$$
C\left(X_{1} ; \cdots ; X_{n}\right)=\left(\sum_{i=1}^{n} H\left(X_{i}\right)\right)-H\left(X_{1}, \ldots, X_{n}\right) .
$$

By using conditional distributions/ conditional entropies, we may define the conditional multivariate total correlation in the obvious way. Note that in the two-variable case, the total correlation is the same as the mutual information $I\left(X_{1} ; X_{2}\right)$.

Theorem 3.2 (Correlation Rounding theorem, [25]). Let $X_{1}, \ldots, X_{n}$ be a collection of $\{ \pm 1\}$-valued random variables. Then, for any $k, \ell \in[n]$, there exists some $t \leq \ell$ such that:

$$
\mathbb{E}_{S \sim\left(\begin{array}{c}
V \\
t
\end{array}\right)} \mathbb{E}_{F \sim\left(\begin{array}{c}
V \\
k
\end{array}\right)}\left[C\left(X_{F} \mid X_{S}\right)\right] \leq \frac{k^{2} \log (2)}{\ell} .
$$

\footnotetext{
${ }^{3}$ This operator may behave very differently from a true expectation. For example, it's possible that $\tilde{\mathbb{E}}\left[f^{2}\right]<0$ for some $f$. The SOS hierarchy is formed by additionally requiring $\tilde{\mathbb{E}}\left[f^{2}\right] \geq 0$ for all low-degree $f$.
}

REMARK 1. The same conclusion holds for general random variables $X_{1}, \ldots, X_{n}$ with the factor $\log 2$ replaced by $\frac{\sum_{i=1}^{n} H\left(X_{i}\right)}{n}$. Also, the guarantee holds for general level $(\ell+k)$-pseudodistributions.

For the reader's convenience, we provide a complete proof of this in the full version of the paper [22], correcting certain errors which have been persistent in the literature.

\subsection{The Sherrington-Kirkpatrick Model and Spin Glass Theory}

The famous Sherrington-Kirkpatrick (SK) spin glass model was introduced in the work [24] as a solvable model of disordered systems. The Gibbs measure of the SK spin glass on $n$ vertices (without external field) is a random probability distribution on $\{ \pm 1\}^{n}$ given by:

$$
\operatorname{Pr}(X=x):=\frac{1}{Z_{n}(\beta)} \exp \left(\frac{\beta}{\sqrt{n}} \sum_{1 \leq i<j \leq n} J_{i j} X_{i} X_{j}\right),
$$

where $J_{i j} \sim N(0,1)$ are i.i.d. standard Gaussians and $\beta$ is a fixed parameter referred to as the inverse temperature. In [24], a prediction, now known as the replica-symmetric prediction, was made for the limiting value of $\frac{1}{n} \log Z_{n}(\beta)$ as $n \rightarrow \infty$. It was soon realized that this prediction could not be correct for all values of $\beta$; finding and understanding the correct prediction led physicists to the development of a sophisticated spin glass theory based upon the non-rigorous replica method ([27]). In particular, physicists showed via the replica method that the SK spin glass exhibits two phases depending on the value of $\beta$ :

(1) Replica Symmetry (RS, $\beta<1$ ). This is the regime where the original prediction for the limiting value of $\frac{1}{n} \log Z_{n}(\beta)$ is correct. Moreover, the Gibbs measure exhibits a number of unusual properties: for example, the marginal law on any small subset of the coordinates converges to a product distribution as $n \rightarrow \infty$ ([33]).

(2) (Full) Replica Symmetry Breaking (fRSB, $\beta>1$ ). In this phase, the limit of $\frac{1}{n} \log Z_{n}(\beta)$ does not have a simple closed form; however, there is a remarkable variational expression for the limiting value known as the Parisi formula. Moreover, the Gibbs measure is understood to be shattered into exponentially many clusters with the geometry of an ultrametric space.

In the replica symmetric phase, the prediction for the limiting value of $\frac{1}{n} \log Z_{n}(\beta)$ was rigorously confirmed by the work of Aizenman, Lebowitz, and Ruelle [2]. Furthermore, they proved their result for general distributions of the $J_{i j}$, giving what is known as a universality result.

Theorem 3.3 ([2]). Let $\epsilon>0$. For the SK spin glass at inverse temperature $\beta<1$,

$$
\operatorname{Pr}\left(\left|\frac{1}{n} \log Z_{n}(\beta)-\left(\log 2+\beta^{2} / 4\right)\right| \geq \epsilon\right) \rightarrow 0
$$

as $n \rightarrow \infty$. Moreover, this also holds if the $J_{i j}$ are i.i.d. samples from any distribution with finite moments, mean 0 and variance 1.

This is the only result we will need from the spin glass literature, although much more is now rigorously known. For an account of more recent developments, including the proofs of the Parisi 
formula and ultrametricity conjecture, we refer the reader to the books $[28,33,34]$.

\section{MEAN-FIELD APPROXIMATION VIA CORRELATION ROUNDING: PROOF OF THEOREM 1.1}

First we recall a couple of lemmas which are essentially used in all works on correlation rounding. Recall that for two probability distributions $P$ and $Q$ on the same finite space $\Omega$, the total variation distance between $P$ and $Q$ is defined by $\operatorname{TV}(P, Q):=$ $\sup _{A \subseteq \Omega}\left|\sum_{a \in A}(P(a)-Q(a))\right|$.

Lemma 4.1 (Lemma 5.1, [5]). Let $X$ and $Y$ be jointly distributed random variables valued in $\{ \pm 1\}$. Let $P_{X}, P_{Y}$ denote the marginal distributions of $X$ and $Y$, and let $P_{X, Y}$ denote their joint distribution. Then,

$$
|\operatorname{Cov}(X, Y)|=2 \operatorname{TV}\left(P_{X, Y}, P_{X} \times P_{Y}\right) .
$$

From this, one can observe the following consequence of correlation rounding:

LEMmA 4.2. Let $X_{1}, \ldots, X_{n}$ be a collection of $\{ \pm 1\}$-valued random variables. Then, for any $\ell \in[n]$, there exists some $S \subset[n]$ with $|S| \leq \ell$ such that:

$$
\mathbb{E}_{X_{S}} \mathbb{E}_{\{u, v\} \in\left(\begin{array}{c}
V \\
2
\end{array}\right)}\left[\operatorname{Cov}\left(X_{u}, X_{v} \mid X_{S}\right)^{2}\right] \leq \frac{8 \log 2}{\ell} .
$$

Proof. We begin by applying Theorem 3.2 with $\ell$; let $S$ denote the resulting set of size at most $\ell$. By Pinsker's inequality, we have

$$
\begin{aligned}
& 2 \operatorname{TV}^{2}\left(\mu\left(X_{u, v} \mid X_{S}=x_{s}\right),\left(\mu\left(X_{u} \mid X_{S}=x_{s}\right) \times \mu\left(X_{v} \mid X_{S}=x_{s}\right)\right)\right) \\
& \quad \leq C\left(X_{u} ; X_{v} \mid X_{S}=x_{s}\right),
\end{aligned}
$$

for any $x_{s} \in\{ \pm 1\}^{|S|}$. Therefore, by taking the expectation on both sides, we get:

$2 \mathbb{E}_{X_{S}} \operatorname{TV}^{2}\left(\mu\left(X_{u, v} \mid X_{S}\right),\left(\mu\left(X_{u} \mid X_{S}\right) \times \mu\left(X_{v} \mid X_{S}\right)\right)\right) \leq C\left(X_{u} ; X_{v} \mid X_{S}\right)$.

By averaging over the choice of $\{u, v\} \in\left(\begin{array}{c}V \\ 2\end{array}\right)$, we get

$$
\begin{aligned}
\mathbb{E}_{E=\{u, v\} \sim\left(\begin{array}{c}
V \\
2
\end{array}\right)} \mathbb{E}_{X_{S}}\left[\operatorname{TV}^{2}\left(\mu\left(X_{u, v} \mid X_{S}\right),\left(\mu\left(X_{u} \mid X_{S}\right) \times \mu\left(X_{v} \mid X_{S}\right)\right)\right)\right] \\
\leq \mathbb{E}_{E \sim\left(\begin{array}{c}
V \\
2
\end{array}\right)}\left[\frac{C\left(X_{E} \mid X_{S}\right)}{2}\right] \\
\leq \frac{2 \log 2}{\ell},
\end{aligned}
$$

where the second inequality follows by the choice of $S$ and Theorem 3.2. Finally, Lemma 4.1 shows that for any $x_{s} \in\{ \pm 1\}^{|S|}$,

$$
\begin{aligned}
& \left|\operatorname{Cov}\left(X_{u}, X_{v} \mid X_{S}=x_{S}\right)\right| \\
& \leq 2 \operatorname{TV}\left(\mu\left(X_{u, v} \mid X_{S}=x_{S}\right),\left(\mu\left(X_{u} \mid X_{S}=x_{S}\right) \times \mu\left(X_{v} \mid X_{S}=x_{S}\right)\right)\right),
\end{aligned}
$$

from which we obtain our desired conclusion:

$$
\mathbb{E}_{X_{S}} \mathbb{E}_{\{u, v\} \in\left(\begin{array}{c}
V \\
2
\end{array}\right)}\left[\operatorname{Cov}\left(X_{u}, X_{v} \mid X_{S}\right)^{2}\right] \leq \frac{8 \log 2}{\ell} .
$$

Finally, we recall the maximum-entropy principle characterizing product distributions:
LEMMA 4.3. Let $\mu$ denote a probability distribution on the finite space $\Omega_{1} \times \cdots \times \Omega_{n}$. Let $v$ denote the product distribution on $\Omega_{1} \times$ $\cdots \times \Omega_{n}$ whose marginal distribution on $\Omega_{i}$ is the same as that of $\mu$ for all $i \in[n]$. Then, $H(\mu) \leq H(v)$.

Proof. This is a direct application of the chain rule and tensorization for entropy. Indeed, let $X:=\left(X_{1}, \ldots, X_{n}\right) \sim \mu$. Then,

$$
H(\mu)=H(X) \leq H\left(X_{1}\right)+\cdots+H\left(X_{n}\right)=H(v) .
$$

We are now ready to prove Theorem 1.1 .

Proof of Theorem 1.1. Let $\epsilon>0$ be some parameter which will be optimized later. We begin by applying Lemma 4.2 with $\ell=1 /\left(\epsilon^{2} \log 2\right)$ (for clarity of exposition, we will omit floors and ceilings since they do not make any essential difference); let $S$ denote the resulting set of size at most $\ell$. Let $\mu$ denote the Boltzmann distribution, and recall that the Gibbs variational principle Eq. (1) states that

$$
\mathcal{F}=\mathbb{E}_{\mu}\left[X^{T} J X\right]+H_{\mu}(X) .
$$

Let $v_{x_{S}}$ denote the product distribution on $\{ \pm 1\}^{n}$ for which $\mathbb{E}_{v_{x_{S}}}\left[X_{i}\right]=\mathbb{E}\left[X_{i} \mid X_{S}=x_{S}\right]$. Then, using the chain rule for entropy, we see that

$$
\begin{aligned}
\mathcal{F} & =\sum_{i<j} J_{i, j} \mathbb{E}_{\mu}\left[X_{i} X_{j}\right]+H_{\mu}(X) \\
& =\sum_{i<j} J_{i, j} \mathbb{E}_{\mu}\left[X_{i} X_{j}\right]+H_{\mu}\left(X \mid X_{S}\right)+H_{\mu}\left(X_{S}\right) \\
& =\mathbb{E}_{x_{S}}\left[\sum_{i<j} J_{i j} \mathbb{E}_{\mu}\left[X_{i} X_{j} \mid X_{S}=x_{S}\right]+H_{\mu}\left(X \mid X_{S}=x_{S}\right)\right]+H_{\mu}\left(X_{S}\right) \\
& \leq \mathbb{E}_{x_{S}}\left[\sum_{i<j} J_{i j} \mathbb{E}_{\mu}\left[X_{i} X_{j} \mid X_{S}=x_{S}\right]+H_{\mu}\left(X \mid X_{S}=x_{S}\right)\right]+1 / \epsilon^{2} \\
& \left.\leq \mathbb{E}_{x_{S}}\left[\sum_{i<j} J_{i j} \mathbb{E}_{\mu}\left[X_{i} X_{j} \mid X_{S}=x_{S}\right]\right]+H_{v_{x_{S}}}(X)\right]+1 / \epsilon^{2},
\end{aligned}
$$

where in the fourth line, we have used that $|S| \leq \ell=1 /\left(\epsilon^{2} \log 2\right)$, and in the last line, we have used Lemma 4.3. From Lemma 4.2 and the Cauchy-Schwarz inequality, it follows that

$$
\begin{aligned}
& \mathbb{E}_{X_{S}} {\left[\sum_{i<j} J_{i j} \mathbb{E}_{\mu}\left[X_{i} X_{j} \mid X_{S}\right]\right] } \\
&= \mathbb{E}_{X_{S}}\left[\sum _ { i < j } J _ { i j } \left(\operatorname{Cov}\left(X_{i}, X_{j} \mid X_{S}=x_{S}\right)\right.\right. \\
&\left.\left.\quad+\mathbb{E}_{\mu}\left[X_{i} \mid X_{S}=x_{S}\right]\left[X_{j} \mid X_{S}=x_{S}\right]\right)\right] \\
&=\sum_{i<j} J_{i, j} \mathbb{E}_{X_{S}}\left[\operatorname{Cov}\left(X_{i}, X_{j} \mid X_{S}\right)\right]+\mathbb{E}_{X_{S}} \sum_{i, j} J_{i, j} \mathbb{E}_{V_{X_{S}}}\left[X_{i} X_{j}\right] \\
& \leq \sqrt{\sum_{i<j} J_{i, j}^{2}} \sqrt{2\left(\begin{array}{c}
|V| \\
2
\end{array}\right) \mathbb{E}_{X_{S}} \mathbb{E}_{E \in\left(\begin{array}{l}
V \\
2
\end{array}\right)}\left[\operatorname{Cov}\left(X_{u}, X_{v} \mid X_{S}\right)^{2}\right]} \\
&+\mathbb{E}_{X_{S} \sum_{i<j} J_{i, j} \mathbb{E}_{V_{X_{S}}}\left[X_{i} X_{j}\right]}
\end{aligned}
$$




$$
\leq 2 \epsilon n\|J\|_{F}+\mathbb{E}_{X_{S}} \sum_{i<j} J_{i, j} \mathbb{E}_{v_{X_{S}}}\left[X_{i} X_{j}\right] .
$$

To summarize, we have shown that

$$
\mathcal{F} \leq \mathbb{E}_{x_{S}}\left[\sum_{i<j} J_{i, j} \mathbb{E}_{v_{x_{S}}}\left[X_{i} X_{j}\right]+H_{v_{x_{S}}}(X)\right]+2 \epsilon n\|J\|_{F}+\frac{1}{\epsilon^{2}} .
$$

In particular, there exists some choice of $x_{S}$, such that with $v:=v_{x_{S}}$, we have

$$
\mathcal{F} \leq\left[\sum_{i<j} J_{i j} \mathbb{E}_{v}\left[X_{i} X_{j}\right]+H_{v}(X)\right]+2 \epsilon n\|J\|_{F}+1 / \epsilon^{2} .
$$

Finally, by setting $\epsilon=\frac{1}{n^{1 / 3}\|J\|_{F}^{1 / 3}}$ we get the desired conclusion:

$$
\mathcal{F} \leq E_{v}\left[\sum_{i<j} J_{i j} X_{i} X_{j}+H(X)\right]+3 n^{2 / 3}\|J\|_{F}^{2 / 3} .
$$

REMARK 2. For the choice of $\epsilon$ in the above proof to make sense, we require that $\ell=1 /\left(\epsilon^{2} \log 2\right) \leq n$, which translates to $\|J\|_{F}^{2 / 3} \leq n^{1 / 3} \log 2$. However, the above bound also holds if $\|J\|_{F}^{2 / 3}>$ $n^{1 / 3} \log 2$ since in this case, our error term equals $3 \log 2 n>2 n$, whereas there is a trivial upper bound of $n \log 2$ on $\mathcal{F}-\mathcal{F}^{*}$, obtained by considering the product distribution supported at the point $\arg \max _{x \in\{ \pm 1\}^{n}}\left\{\sum_{i j} J_{i j} x_{i} x_{j}\right\}$.

REMARK 3. The above proof shows that for the product measure $v:=v_{x_{S}}, \mathcal{F}_{v}$ is close to $\mathcal{F}$. This shows indirectly, by considering the maximizer of $\mathcal{F}^{*}$, that there exists a product distribution with marginals that are an exact solution to the mean-field equation $x=\tanh ^{\otimes n}(J x)$ which is close to the Gibbs distribution in KL distance. In [22], we show that the marginals output by correlation rounding are already an approximate solution to the mean-field equation, given an additional assumption on $J$.

\section{MEAN-FIELD APPROXIMATION FOR $k$-MRFS}

In this section, we prove a much more general bound for mean-field approximation, extending our result Theorem 1.1 to order $k$ Markov random fields (MRFs) over general finite alphabets. Our bound has only a mild dependence on the alphabet size $q$ and is tight for every fixed $k, q$.

Definition 5.1. An order $k$ Markov random field ( $k$-MRF) on $n$ vertices over the finite alphabet $\Sigma$ is a probability distribution on the space $\Sigma^{n}$ of the form

$$
\operatorname{Pr}(X=x)=\frac{1}{Z} e^{f(x)+h(x)}
$$

where the interaction term $f(x)$ can be written as a sum of hyperedge potentials on hyperedges of size $k$ i.e.

$$
f(x)=\sum_{E \subseteq[n],|E|=k} f_{E}\left(x_{E}\right),
$$

and the external field $h(x)$ is the sum of the external fields at each vertex i.e.

$$
h(x)=\sum_{i=1}^{n} h_{i}\left(x_{i}\right)
$$

In analogy with the Ising model case, we will denote $\sup _{x_{E}}\left|f_{E}\left(x_{E}\right)\right|$ by $\left\|f_{E}\right\|_{\infty}$ and $\sum_{E \subseteq[n],|E|=k}\left\|f_{E}\right\|_{\infty}^{2}$ by $\|J\|_{F}^{2}$. The exact same proof as the Ising case gives the following variational principle for the free energy $\mathcal{F}:=\log Z$ :

$$
\mathcal{F}=\sup _{\mu}\left[\mathbb{E}_{\mu}[f(x)+h(x)]+H(\mu)\right]
$$

where the supremum ranges over all probability distributions on $\Sigma^{n}$. By restricting the variational problem to product distributions over $\Sigma^{n}$, we obtain the variational free energy $\mathcal{F}^{*}$ as before.

TheOREM 5.2. For any $k-M R F$ on $n$ vertices over an alphabet of size $q$,

$$
\mathcal{F}-\mathcal{F}^{*} \leq 3\left(\frac{k \log q}{\sqrt{k !}} n^{k / 2}\|J\|_{F}\right)^{2 / 3}
$$

The proof of this theorem is essentially the same as that of Theorem 1.1 with appropriate modifications. We will need the following simple lemma.

LEMMA 5.3. Let $\mu$ and $v$ are two probability distributions on the same space $\Omega$. Then for any function $f: \Omega \rightarrow \mathbb{R}$ such that $|f(X)| \leq M$ a.s. under both $\mu$ and $v$, we have

$$
\left|\mathbb{E}_{X \sim \mu}[f(X)]-\mathbb{E}_{Y \sim v}[f(Y)]\right| \leq 2 M \operatorname{TV}(\mu, v) .
$$

Proof. By a standard characterization of TV, we can couple $X$ and $Y$ so that $\operatorname{Pr}(X \neq Y)=\operatorname{TV}(\mu, v)$. Since $|f(X)-f(Y)| \leq 2 M$ a.s, we are done.

Proof of Theorem 5.2. Let $\epsilon>0$ be some parameter which will be optimized later. We begin by applying Theorem 3.2 with $\ell=1 /\left(\epsilon^{2} \log q\right)$; let $S$ be the resulting set of size at most $\ell$. Let $\mu$ denote the Boltzmann distribution. For each assignment $x_{S} \in \Sigma^{|S|}$ to the variables in $S$, let $v_{x_{S}}$ denote the product measure on $\Sigma^{n}$ for which $\mathbb{E}_{v_{x_{S}}}\left[X_{i}\right]=\mathbb{E}\left[X_{i} \mid X_{S}=x_{S}\right]$. Then, using the variational principle, the same computation as in the binary Ising model case shows that

$$
\mathcal{F} \leq \mathbb{E}_{x_{S}}\left[\mathbb{E}_{\mu}\left[f(X) \mid X_{S}=x_{S}\right]+\mathbb{E}_{v_{x_{S}}}[h(X)]+H_{v_{x_{S}}}(X)\right]+\ell \log q .
$$

As before, we decompose the first term as

$$
\begin{aligned}
\mathbb{E}_{x_{S}}\left[\mathbb { E } _ { \mu } \left[f(X) \mid X_{S}\right.\right. & \left.=x_{S}\right]=\mathbb{E}_{x_{S}}\left[\mathbb{E}_{v_{x_{S}}}[f(X)]\right] \\
& +\mathbb{E}_{x_{S}}\left[\left[\mathbb{E}_{\mu}\left[f(X) \mid X_{S}=x_{S}\right]-\mathbb{E}_{v_{x_{S}}}[f(X)]\right]\right] .
\end{aligned}
$$

Since $f(X)=\sum_{E \in\left(\begin{array}{c}{[n]} \\ k\end{array}\right)} f\left(X_{E}\right)$, it follows by Lemma 5.3 that

$$
\begin{array}{r}
\mathbb{E}_{x_{S}}\left|\mathbb{E}_{\mu}\left[f(X) \mid X_{S}=x_{S}\right]-\mathbb{E}_{v_{x_{S}}}[f(X)]\right| \leq \\
2\left(\begin{array}{c}
n \\
k
\end{array}\right) \mathbb{E}_{x_{S}} \mathbb{E}_{E \sim\left(\begin{array}{c}
{[n]} \\
k
\end{array}\right)}\left[\left\|f_{E}\right\|_{\infty} \operatorname{TV}\left(\left.\left(\mu \mid X_{S}=x_{S}\right)\right|_{X_{E}}, v_{x_{S}} \mid X_{E}\right)\right] .
\end{array}
$$

By the Cauchy-Schwarz inequality, the right hand side is bounded by

$$
2 \sqrt{\left(\begin{array}{l}
n \\
k
\end{array}\right)}\|J\|_{F} \sqrt{\mathbb{E}_{X_{S}} \mathbb{E}_{E \sim\left(\begin{array}{c}
{[n]} \\
k
\end{array}\right)} \operatorname{TV}^{2}\left(\left.\left(\mu \mid X_{S}=x_{S}\right)\right|_{X_{E}},\left.v_{x_{S}}\right|_{X_{E}}\right)},
$$


whereas by Pinsker's inequality and the choice of $S$, we have

$$
\begin{gathered}
\sqrt{\mathbb{E}_{X_{S}} \mathbb{E}_{E \sim\left(\begin{array}{c}
{[n]} \\
k
\end{array}\right)} \operatorname{TV}^{2}\left(\left.\left(\mu \mid X_{S}=x_{S}\right)\right|_{X_{E}}, v_{X_{S}} \mid X_{E}\right)} \leq \\
\sqrt{\mathbb{E}_{E \sim\left(\begin{array}{c}
{[n]} \\
k
\end{array}\right)} C\left(\left.\left(\mu \mid X_{S}\right)\right|_{X_{E}},\left.v_{X_{S}}\right|_{X_{E}}\right)} \leq \frac{k \sqrt{\log q}}{\sqrt{\ell}} .
\end{gathered}
$$

To summarize, there exists some $x_{S}$ such that the associated product distribution $v:=v_{x_{S}}$ satisfies

$$
\mathcal{F} \leq \mathbb{E}_{v}[f(x)+h(x)]+H(v)+2 k \epsilon \sqrt{\left(\begin{array}{l}
n \\
k
\end{array}\right)}\|J\|_{F} \log q+\frac{1}{\epsilon^{2}} .
$$

Using $\left(\begin{array}{l}n \\ k\end{array}\right) \leq n^{k} / k$ ! and optimizing the value of $\epsilon$ completes the proof.

In the full version of this paper [22], we discuss the tightness of Theorem 5.2 .

\section{CORRELATION ROUNDING IS TIGHT FOR SPIN GLASSES: PROOF OF THEOREM 1.3}

We define the following universal constant, which we already know an upper bound on by Theorem 2.3:

$$
\kappa_{*}:=\limsup _{t \rightarrow \infty} \sup _{\substack{\mu \in \mathcal{P}\left(\{ \pm 1\}^{n}\right) \\
n \geq t}} \min _{S:|S| \leq t} \sqrt{t} \mathbb{E}_{(i, j) \sim\left(\begin{array}{c}
{[n]} \\
2
\end{array}\right)}\left[\left|\operatorname{Cov}\left(X_{i}, X_{j} \mid X_{S}\right)\right|\right] .
$$

If Conjecture 2.4 were true, then we would have $\kappa_{*}=0-$ indeed, the conjecture says that the expected conditional covariance decays like $O(1 / t)$, even for a random choice of the conditioning set $S$. We will instead show an explicit positive lower bound on $\kappa_{*}$, thereby disproving the conjecture.

We begin by proving a variant of Theorem 1.1, which gives a bound on the error of the mean-field approximation in terms of the constant $\kappa_{*}$.

LEMMA 6.1. Let $\left\{J_{n}\right\}_{n \geq 1}$ be a sequence of Ising models indexed by the number of vertices. Let $\mathcal{F}_{n}$ (resp. $\mathcal{F}_{n}^{*}$ ) denote the free energy (resp. variational free energy) of $J_{n}$. Suppose that $\kappa_{*}^{2} \lim \sup _{n \rightarrow \infty} n\left\|J_{n}\right\|_{\infty}^{2}<$ 16. Then,

$$
\limsup _{n \rightarrow \infty} \frac{\mathcal{F}_{n}-\mathcal{F}_{n}^{*}}{n^{4 / 3}\left\|J_{n}\right\|_{\infty}^{2 / 3}} \leq \frac{3}{\sqrt[3]{4}} \kappa_{*}^{2 / 3} .
$$

Proof. Let $\left\{t_{n}\right\}_{n \geq 1}$ be a sequence of natural numbers going to infinity, which will be specified later; our choice will be such that $t_{n} \leq n$ for all $n$. For the Ising model $J_{n}$, let

$$
S_{n}:=\underset{S \subseteq[n],|S| \leq t_{n}}{\arg \min } \sqrt{t_{n}} \mathbb{E}_{(i, j) \sim\left(\begin{array}{c}
{[n]} \\
2
\end{array}\right)}\left[\left|\operatorname{Cov}\left(X_{i}, X_{j} \mid X_{S}\right)\right|\right],
$$

and let $\kappa_{n}$ denote the minimum value i.e. the value of the objective corresponding to $S_{n}$. By repeating the first part of the proof of Theorem 1.1, we get

$$
\begin{aligned}
& \mathcal{F}_{n} \leq\left.\mathbb{E}_{x_{S_{n}}}\left[\sum_{i j}\left(J_{n}\right)_{i j} \mathbb{E}_{\mu}\left[X_{i} X_{j} \mid X_{S_{n}}=x_{S_{n}}\right]\right]+H_{v_{x_{S_{n}}}}(X)\right]+t_{n} \\
& \leq \sum_{i, j}\left(J_{n}\right)_{i, j}\left[\operatorname{Cov}\left(X_{i}, X_{j} \mid X_{S_{n}}\right)\right] \\
& \quad+\mathbb{E}_{x_{S_{n}}}\left[\sum_{i, j}\left(J_{n}\right)_{i, j} \mathbb{E}_{v_{x_{S_{n}}}}\left[X_{i} X_{j}\right]+H_{v_{x_{S_{n}}}}(X)\right]+t_{n} .
\end{aligned}
$$

As opposed to the proof of Theorem 1.1 where we used the CauchySchwarz inequality, here we simply estimate the first term by

$$
\sum_{i, j}\left(J_{n}\right)_{i, j}\left[\left|\operatorname{Cov}\left(X_{i}, X_{j} \mid X_{S_{n}}\right)\right|\right] \leq 2\left(\begin{array}{l}
n \\
2
\end{array}\right) \frac{\kappa_{n}\left\|J_{n}\right\|_{\infty}}{\sqrt{t_{n}}} .
$$

Finally, set

$$
t_{n}=\min \left\{\frac{n^{4 / 3} \kappa_{n}^{2 / 3}\left\|J_{n}\right\|_{\infty}^{2 / 3}}{\sqrt[3]{4}}, n\right\}
$$

note that $t_{n}<n$ for all sufficiently large $n$ by assumption, along with the fact that $\lim \sup _{n \rightarrow \infty} \kappa_{n} \leq \kappa_{*}$. It follows that for all $n$ sufficiently large,

$$
\mathcal{F}_{n}-\mathcal{F}_{n}^{*} \leq \frac{3}{\sqrt[3]{4}} n^{4 / 3}\left\|J_{n}\right\|_{\infty}^{2 / 3} \kappa_{n}^{2 / 3}
$$

dividing both sides by $n^{4 / 3}\left\|J_{n}\right\|_{\infty}^{2 / 3}$, taking the lim sup as $n \rightarrow \infty$, and using $\lim \sup _{n \rightarrow \infty} \kappa_{n} \leq \kappa_{*}$ yields the desired conclusion.

To complete the proof of Theorem 1.3, we will exhibit a sequence of Ising models $J_{n}$ for which $\lim \sup _{n \rightarrow \infty} n\left\|J_{n}\right\|_{\infty}^{2}$ is finite and $\lim \sup _{n \rightarrow \infty}\left(\mathcal{F}_{n}-\mathcal{F}_{n}^{*}\right) /\left(n^{4 / 3}\left\|J_{n}\right\|_{\infty}^{2 / 3}\right)$ is positive. Specifically, we will show that this is true for a 'typical' growing sequence of the Rademacher SK-spin glass. First, we need the following lemma.

Lemma 6.2. Fix $\beta \in[0,1 / 2)$. Let $\mathcal{F}_{n}(\beta)$ denote the (random) free energy of the SK spin glass on $n$ vertices with parameter $\beta$, and let $\mathcal{F}_{n}^{*}(\beta)$ denote its variational free energy. Then,

$$
\mathcal{F}_{n}(\beta)-\mathcal{F}_{n}^{*}(\beta) \geq n \beta^{2} / 4-o(n)
$$

asymptotically almost surely (a.a.s) i.e. with probability going to 1 as $n \rightarrow \infty$. This holds under the same universality regime as Theorem 3.3.

Proof. We prove this by calculating $\mathcal{F}_{n}(\beta)$ and $\mathcal{F}_{n}^{*}(\beta)$. Since $\beta<1$, we know from Theorem 3.3 that a.a.s.

$$
\frac{\mathcal{F}_{n}(\beta)}{n}=\log 2+\frac{\beta^{2}}{4}+o_{n}(1)
$$

It remains to compute $\mathcal{F}_{n}^{*}(\beta)$. By definition,

$$
\mathcal{F}_{n}^{*}(\beta)=\sup _{x \in[-1,1]^{n}}\left(\frac{\beta}{2} x^{T} J x+\sum_{i} H\left(\frac{1+x_{i}}{2}\right)\right) .
$$

We claim that a.a.s., this optimization problem is concave - indeed, direct calculation shows that for all $x \in[-1,1]$

$$
\frac{d^{2}}{d x^{2}} H\left(\frac{1+x}{2}\right) \leq-1,
$$

whereas Wigner's semicircle law ([36]) shows that

$$
\|J\| \leq 2+o_{n \rightarrow \infty}(1)
$$

a.a.s. Since the Hessian of first term is $J$, this proves the claim since $0 \leq \beta<1 / 2$.

Finally, since the gradient of the objective function

$$
\frac{\beta}{2} x^{T} J x+\sum_{i} H\left(\frac{1+x_{i}}{2}\right)
$$

clearly vanishes at the point $x_{i}=0$ for all $i \in[n]$, it follows that this point is the global maximizer a.a.s, so that $\mathcal{F}_{n}^{*}(\beta)=n \log 2$ a.a.s. 
By combining the previous two lemmas, we can prove the following theorem which, in particular, implies Theorem 1.3.

THEOREM 6.3. Let $\kappa_{*}$ be the universal constant defined at the start of this section.

$$
\kappa_{*} \geq \frac{\sqrt{27}}{16} .
$$

Proof. From Lemma 6.2 applied to the Rademacher SK spin glass with parameter $\beta \in[0,1 / 2)$ i.e. $\left(J_{n}\right)_{i j}= \pm \beta / \sqrt{n}$ independently with probability $1 / 2$, we obtain a sequence of Ising models $\left\{J_{n}\right\}_{n \geq 1}$ indexed by the number of vertices for which the following holds:

- $\left\|J_{n}\right\|_{\infty}=\frac{\beta}{\sqrt{n}}$ i.e. $\lim \sup _{n \rightarrow \infty} n\left\|J_{n}\right\|_{\infty}^{2}=\beta^{2}$

- $\lim \sup _{n \rightarrow \infty} \frac{\mathcal{F}_{n}-\mathcal{F}_{n}^{*}}{n \beta^{2}} \geq \frac{1}{4}$ i.e. $\lim \sup _{n \rightarrow \infty} \frac{\mathcal{F}_{n}-\mathcal{F}_{n}^{*}}{n^{4 / 3}\left\|J_{n}\right\|_{\infty}^{2 / 3}} \geq$ $\frac{\beta^{4 / 3}}{4}$.

In view of Lemma 6.1, there are two possibilities:

- $\kappa_{*}^{2} \beta^{2} \geq 16$ for some $\beta \in[0,1 / 2)$, so that $\kappa_{*} \geq 4$, or

- $\kappa_{*}^{2} \beta^{2}<16$ for all $\beta \in[0,1 / 2]$, in which case we have

$$
\frac{\beta^{4 / 3}}{4} \leq \frac{3}{\sqrt[3]{4}} \kappa_{*}^{2 / 3}
$$

for all $\beta \in[0,1 / 2)$, so that $\kappa_{*} \geq \sqrt{27} / 16$.

\section{ALGORITHMIC RESULTS: PROOF OF THEOREM 1.2}

We now show how to go from the proof of our bounds on the quality of mean-field approximation to concrete algorithms; this is a relatively straightforward application of the Sherali-Adams relaxation. The only serious difficulty is to find a good proxy for the entropy that is suitable for use with pseudo-distributions; this was solved in [31] by introducing the following pseudo-entropy functional for level $(r+1)$ pseudo-distributions:

$$
\tilde{H}_{r}(\mu)=\min _{S:|S| \leq r}\left[H\left(X_{S}\right)+\sum_{i} H\left(X_{i} \mid X_{S}\right)\right] .
$$

By the chain rule for entropy, we see that for any $r$ and for any true probability distribution $\mu, H(\mu) \leq \tilde{H}_{r}(\mu)$. Moreover, essentially the standard proof of the concavity of entropy shows that for any $r$, $\tilde{H}_{r}(\mu)$ is a concave function of the pseudo-distribution $\mu$ (Lemma 8 of [31]). Then, we can write the Sherali-Adams relaxation to Eq. (6) as

$$
\mathcal{F}_{S A, r+k}:=\max _{\mu \in S A_{r+k}} \tilde{E}[f(X)+h(X)]+\tilde{H}_{r}(\mu) .
$$

Note that by considering the Boltzmann distribution $\mu$ in the above optimization problem, and using that $H(\mu) \leq \tilde{H}_{r}(\mu)$, it follows that $\mathcal{F}_{S A, r+k} \geq \mathcal{F}$.

Combining this relaxation with correlation rounding gives Algorithm SA-MEANFIELD for finding good mean-field solutions.

REMARK 4. Instead of searching over all $S \subseteq[n]$ with $|S| \leq r$, we may greedily select $S$ vertex by vertex, stopping when the average total correlation $\mathbb{E}_{E}\left[C\left(X_{E} \mid X_{S}\right)\right]$ satisfies the guarantee of Theorem 3.2. That this works follows from a slightly modified analysis of correlation rounding.

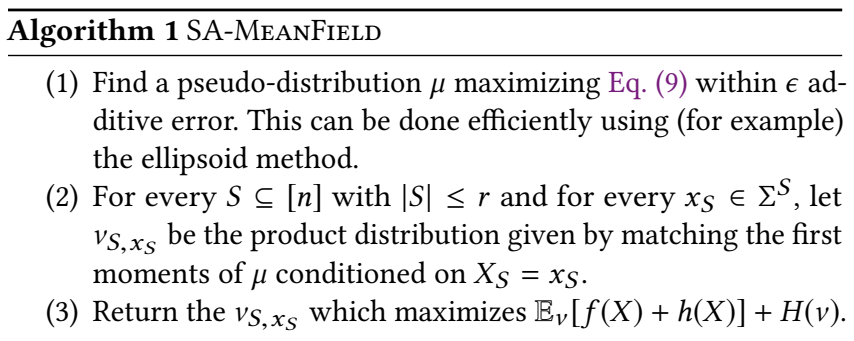

Theorem 7.1. Let $H(p)$ denote the entropy of $\operatorname{Ber}(p)$. We have the following running time and performance guarantees for Algorithm SA-MEANFIELD.

(1) The running time is

$$
{ }_{2} O(n H((r+k) / n)+(r+k) \log q)+\operatorname{poly} \log (1 / \epsilon) .
$$

(2) The product distribution $v$ returned by the algorithm satisfies

$$
0 \leq \mathcal{F}-\mathcal{F}_{v} \leq \sqrt{\frac{4 \log q}{r}} \frac{k n^{k / 2}\|J\|_{F}}{\sqrt{k !}}+r \log q+\epsilon,
$$

where

$$
\mathcal{F}_{v}:=\mathbb{E}_{v}[f(X)+h(X)]+H(v) .
$$

(3) We also have the following guarantee for the pseudodistribution $\mu$ computed in the first step:

$$
0 \leq \mathcal{F}_{S A, r+k}(\mu)-\mathcal{F} \leq \sqrt{\frac{4 \log q}{r}} \frac{k n^{k / 2}\|J\|_{F}}{\sqrt{k !}}+\epsilon,
$$

where

$$
\mathcal{F}_{S A, r+k}(\mu):=\tilde{E}_{\mu}[f(X)+h(X)]+\tilde{H}_{r}(\mu) .
$$

Proof. The runtime is dominated by the first step, where we solve a convex program with at most $q^{r+k}\left(\begin{array}{c}n \\ r+k\end{array}\right)$ many variables and poly $\left(q^{r+k}\left(\begin{array}{c}n \\ r+k\end{array}\right)\right)$ many LP constraints. Therefore, by standard guarantees for the ellipsoid method [17] we can solve Eq. (9) within $\epsilon$ additive error in time poly $\left(q^{r+k}\left(\begin{array}{c}n \\ r+k\end{array}\right), \log (1 / \epsilon)\right)$. Using the standard bound (which follows from sub-additivity of entropy)

$$
\log \left(\begin{array}{c}
n \\
r+k
\end{array}\right) \leq n H\left(\frac{r+k}{n}\right)
$$

this quantity is at most poly $\left(2^{O} O(n H((r+k) / n)+(r+k) \log q), \log (1 / \epsilon)\right)$. Finally, we use the AM-GM inequality to separate the ${ }_{2} O(n H((r+k) / n)+(r+k) \log q)$ term in the bound.

For 2., note that $0 \leq \mathcal{F}-\mathcal{F}_{v}$ follows from the Gibbs variational principle, so we only need to show the right inequality. We will deduce this from the stronger (since $\mathcal{F}_{S A, r+2} \geq \mathcal{F}$ ) statement

$$
\mathcal{F}_{S A, r+2}-\mathcal{F}_{v} \leq \sqrt{\frac{4 \log q}{r}} \frac{k n^{k / 2}\|J\|_{F}}{\sqrt{k !}}+r \log q+\epsilon,
$$

which itself follows from

$$
\mathcal{F}_{S A, r+2}(\mu)-\mathcal{F}_{v} \leq \sqrt{\frac{4 \log q}{r}} \frac{k n^{k / 2}\|J\|_{F}}{\sqrt{k !}}+r \log q,
$$

where $\mu$ is the $r+2$ pseudo-distribution returned in the first step. Now, note that Eq. (11) follows by exactly the same proof as for 
Theorem 5.2 (in particular, Eq. (7)) using the fact that an $r+k$ pseudodistribution suffices to give the correlation rounding guarantee on sets of size at most $r$, and recalling that in Eq. (7), $\epsilon=1 / \sqrt{r \log q}$.

Finally, 3. follows from Eq. (11), noting additionally that we can avoiding losing the term $r \log q$ (equivalently, the term $1 / \epsilon^{2}$ in Eq. (7)), if we round instead to the mixture of product distributions given by $\sum_{x_{S}} P\left(x_{S}\right) v_{S, x_{S}}$.

In particular, we obtain the following more general and precise version of Theorem 1.2.

Corollary 7.2. Fix $k$ and $q$. If $\left\|J_{n}\right\|_{F} \leq c_{k, q} f(n) n^{3 / 2-k / 2}$, where $f(n) \rightarrow 0$ as $n \rightarrow \infty$ and $c_{k, q}>0$ is some constant depending only on $k$ and $q$, then $\mathcal{F}_{n}$ can be approximated to within $\sqrt{f(n)} n$ additive error in (sub-exponential) time $2^{-O(n \sqrt{f(n)} \log f(n))}$ by Algorithm SAMEANFIELD. Moreover, the algorithm outputs a product distribution achieving this approximation.

In the full version of this paper, we also discuss how to obtain faster randomized algorithms for approximating the free energy on dense instances by combining the above with the main result in [21].

\section{CONCLUSION}

We presented a unified perspective on two major variational approaches to calculating the free energy that hitherto seemed completely disparate: mean-field approximations and convex relaxations. This view has both analytic benefits (we derived bounds on the quality of mean-field approximations) and algorithmic benefits (we derived algorithms for approximating the free energy up to the intractability limit).

We conclude with several open problems:

(1) As mentioned earlier, there is a straightforward example showing that up to a constant, the exponent $\frac{2}{3}$ is optimal in Theorem 1.1 for the natural univariate quantity $\left(n\|J\|_{F}\right)$ However, this example does not rule out other bounds of the form $O\left(n^{1-\alpha}\|J\|_{F}^{2 \alpha}\right)$ for $\alpha \in[0,1]$. As there is always a trivial bound $O(n)$ for the mean-field approximation (consider the optimal point-mass distribution), we may assume that $\|J\|_{F}=o\left(n^{1 / 2}\right)$ and ask about the supremum of all $\alpha$ such that an upper bound of this form holds. The Curie-Weiss model at critical temperature shows that we cannot take $\alpha$ to be 0 without introducing additional logarithmic factors in the upper bound. Other than this, we have unfortunately not been able to rule out any other values of $\alpha \in(0,1 / 3]$.

(2) It's possible that the fRSB phase of the SK spin glass is more difficult to correlation-round than the RS phase. Indeed, the landscape picture for the fRSB phase seems like a natural obstruction to correlation rounding and was what originally motivated us to consider spin glasses. Is one of these spin glass models extremal, in the sense that they can be used to get the optimal value of $\kappa_{*}$ ? If not, what do the extremal distributions look like?

(3) How many rounds do convex hierarchies (Sherali-Adams, Sum-of-Squares) need to correctly estimate the value of the free energy and ground state of the SK spin glass? (By computing the ground state, we mean to drop the entropy and just consider the MAX-QP problem.) Are $\Omega(n)$ rounds required?

\section{ACKNOWLEDGMENTS}

We would like to thank Elchanan Mossel for stimulating discussions and Ankur Moitra for providing valuable feedback on the paper.

\section{REFERENCES}

[1] Nir Ailon and Noga Alon. 2007. Hardness of fully dense problems. Information and Computation 205, 8 (2007), 1117-1129.

[2] Michael Aizenman, Joel L Lebowitz, and David Ruelle. 1987. Some rigorous results on the Sherrington-Kirkpatrick spin glass model. Communications in mathematical physics 112, 1 (1987), 3-20.

[3] Sarah R Allen and Ryan O'Donnell. 2015. Conditioning and covariance on caterpillars. In Information Theory Workshop (ITW), 2015 IEEE. IEEE, 1-5.

[4] James Anderson and Carsten Peterson. 1987. A Mean Field Theory Learning Algorithm for Neural Networks. Complex Systems 1 (1987), 995-1019. Issue 5.

[5] Boaz Barak, Prasad Raghavendra, and David Steurer. 2011. Rounding semidefinite programming hierarchies via global correlation. In Foundations of Computer Science (FOCS), 2011 IEEE 52nd Annual Symposium on. IEEE, 472-481.

[6] Anirban Basak and Sumit Mukherjee. 2017. Universality of the mean-field for the Potts model. Probability Theory and Related Fields 168, 3-4 (2017), 557-600.

[7] Christian Borgs, Jennifer T Chayes, László Lovász, Vera T Sós, and Katalin Vesztergombi. 2012. Convergent sequences of dense graphs II. Multiway cuts and statistical physics. Annals of Mathematics 176, 1 (2012), 151-219.

[8] Amin Coja-Oghlan and Will Perkins. 2017. Bethe states of random factor graphs. arXiv preprint arXiv:1709.03827 (2017).

[9] Fernandez de la Vega and Marek Karpinski. 2006. Approximation Complexity of Nondense Instances of MAX-CUT. Electronic Colloquium on Computational Complexity (2006). TR06-101.

[10] Wenceslas Fernandez de la Vega and Claire Kenyon-Mathieu. 2007. Linear programming relaxations of maxcut. In Proceedings of the eighteenth annual ACM-SIAM symposium on Discrete algorithms. Society for Industrial and Applied Mathematics, 53-61.

[11] Amir Dembo and Andrea Montanari. 2010. Ising models on locally tree-like graphs. Ann. Appl. Probab. 20, 2 (2010), 565-592. https://doi.org/10.1214/ 09-AAP627

[12] Ronen Eldan. 2016. Gaussian-width gradient complexity, reverse log-Sobolev inequalities and nonlinear large deviations. arXiv preprint arXiv:1612.04346 (2016).

[13] Ronen Eldan and Renan Gross. 2018. Decomposition of mean-field Gibbs distributions into product measures. Electronic fournal of Probability 23 (2018).

[14] Richard S Ellis and Charles M Newman. 1978. The statistics of Curie-Weiss models. Fournal of Statistical Physics 19, 2 (1978), 149-161.

[15] Dimitris Fotakis, Michael Lampis, and Vangelis Th Paschos. 2016. Sub-exponential Approximation Schemes for CSPs: From Dense to Almost Sparse. In 33rd Symposium on Theoretical Aspects of Computer Science.

[16] Alan Frieze and Ravi Kannan. 1999. Quick approximation to matrices and applications. Combinatorica 19, 2 (1999), 175-220.

[17] Martin Grötschel, László Lovász, and Alexander Schrijver. 2012. Geometric algorithms and combinatorial optimization. Springer Science \& Business Media.

[18] Venkatesan Guruswami and Ali Kemal Sinop. 2011. Lasserre hierarchy, higher eigenvalues, and approximation schemes for graph partitioning and quadratic integer programming with PSD objectives. In Foundations of Computer Science (FOCS), 2011 IEEE 52nd Annual Symposium on. IEEE, 482-491.

[19] Dmitry Ioffe and Yvan Velenik. 2000. A note on the decay of correlations under $\delta$-pinning. Probability theory and related fields 116, 3 (2000), 379-389.

[20] Vishesh Jain, Frederic Koehler, and Elchanan Mossel. 2018. The Mean-Field Approximation: Information Inequalities, Algorithms, and Complexity. In Conference on Learning Theory (COLT).

[21] Vishesh Jain, Frederic Koehler, and Elchanan Mossel. 2018. The Vertex Sample Complexity of Free Energy is Polynomial. In Conference on Learning Theory (COLT).

[22] Vishesh Jain, Frederic Koehler, and Andrej Risteski. 2018. Mean-field approximation, convex hierarchies, and the optimality of correlation rounding: a unified perspective. arXiv preprint arXiv:1808.07226 (2018).

[23] Michael I Jordan, Zoubin Ghahramani, Tommi S Jaakkola, and Lawrence K Saul. 1999. An introduction to variational methods for graphical models. Machine learning 37, 2 (1999), 183-233.

[24] S Kirkpatrick and D Sherrington. 1975. Solvable model of a spin-glass. Phys. Rev. Lett 35, 26 (1975), 1792-1796.

[25] Pasin Manurangsi and Prasad Raghavendra. 2017. A Birthday Repetition Theorem and Complexity of Approximating Dense CSPs. In Proceedings of ICALP, Vol. 80. Schloss Dagstuhl-Leibniz-Zentrum fuer Informatik. 
[26] Claire Mathieu and Warren Schudy. 2008. Yet Another Algorithm for Dense Max Cut: Go Greedy. In Proceedings of the Nineteenth Annual ACM-SIAM Symposium on Discrete Algorithms (SODA '08). Society for Industrial and Applied Mathematics, Philadelphia, PA, USA, 176-182. http://dl.acm.org/citation.cfm?id=1347082. 1347102

[27] Marc Mézard, Giorgio Parisi, and Miguel Virasoro. 1987. Spin glass theory and beyond: An Introduction to the Replica Method and Its Applications. Vol. 9. World Scientific Publishing Company.

[28] Dmitry Panchenko. 2013. The Sherrington-Kirkpatrick model. Springer Science \& Business Media.

[29] Giorgio Parisi. 1988. Statistical field theory. New York: Addison-Wesley.

[30] Prasad Raghavendra and Ning Tan. 2012. Approximating CSPs with global cardinality constraints using SDP hierarchies. In Proceedings of the twenty-third annual ACM-SIAM symposium on Discrete Algorithms. Society for Industrial and Applied Mathematics, 373-387.

[31] Andrej Risteski. 2016. How to calculate partition functions using convex programming hierarchies: provable bounds for variational methods. In COLT.

[32] Allan Sly and Nike Sun. 2012. The computational hardness of counting in twospin models on d-regular graphs. In Foundations of Computer Science (FOCS), 2012 IEEE 53rd Annual Symposium on. IEEE, 361-369.
[33] Michel Talagrand. 2011. Mean field models for spin glasses. Volume I. Ergebnisse der Mathematik und ihrer Grenzgebiete. 3. Folge. A Series of Modern Surveys in Mathematics [Results in Mathematics and Related Areas. 3rd Series. A Series of Modern Surveys in Mathematics], Vol. 54. Springer-Verlag, Berlin. xviii+485 pages. Basic examples.

[34] Michel Talagrand. 2011. Mean Field Models for Spin Glasses. Volume II: Advanced Replica-Symmetry and Low Temperature. Ergebnisse der Mathematik und ihrer Grenzgebiete. 3. Folge. A Series of Modern Surveys in Mathematics [Results in Mathematics and Related Areas. 3rd Series. A Series of Modern Surveys in Mathematics], Vol. 55. Springer, Heidelberg (2011b). ISBN.

[35] Martin J. Wainwright and Michael I. Jordan. 2008. Graphical models, exponential families, and variational inference. Foundations and Trends in Machine Learning 1(1-2) (2008), 1-305.

[36] Eugene P Wigner. 1958. On the distribution of the roots of certain symmetric matrices. Annals of Mathematics (1958), 325-327.

[37] Yuichi Yoshida and Yuan Zhou. 2014. Approximation schemes via Sherali-Adams hierarchy for dense constraint satisfaction problems and assignment problems. In Proceedings of the 5th conference on Innovations in theoretical computer science. ACM, 423-438. 